\title{
Article
}

\section{Observations of cold dust in nearby elliptical galaxies}

Leeuw, LL, Sansom, Anne E, Robson, El and Haas, M

Available at http://clok.uclan.ac.uk/206/

Leeuw, LL, Sansom, Anne E ORCID: 0000-0002-2782-7388, Robson, El and Haas, M (2004) Observations of cold dust in nearby elliptical galaxies. The Astrophysical Journal, 612 (2). pp. 837-847. ISSN 0004-637X

It is advisable to refer to the publisher's version if you intend to cite from the work. http://dx.doi.org/10.1086/422795

For more information about UCLan's research in this area go to

http://www.uclan.ac.uk/researchgroups/ and search for < name of research Group>.

For information about Research generally at UCLan please go to http://www.uclan.ac.uk/research/

All outputs in CLoK are protected by Intellectual Property Rights law, including Copyright law. Copyright, IPR and Moral Rights for the works on this site are retained by the individual authors and/or other copyright owners. Terms and conditions for use of this material are defined in the policies page.

\section{CLoK}

Central Lancashire online Knowledge www.clok.uclan.ac.uk 


\title{
OBSERVATIONS OF COLD DUST IN NEARBY ELLIPTICAL GALAXIES
}

\author{
Lerothodi L. Leeuw, ${ }^{1}$ Anne E. Sansom, ${ }^{2}$ E. Ian Robson, ${ }^{3}$ Martin Haas, ${ }^{4}$ and Nario Kuno ${ }^{5}$ \\ Received 2003 October 6; accepted 2004 May 25
}

\begin{abstract}
Spectral energy distribution (SED) analyses that include new millimeter to far-infrared (FIR) observations obtained with continuum instruments on the Nobeyama and James Clerk Maxwell Telescopes and the Infrared Space Observatory are presented for seven nearby $(<45 \mathrm{Mpc})$ FIR-bright elliptical galaxies. These are analyzed together with archival FIR and shortwave radio data obtained from the NASA/IPAC Extragalactic Database (NED). The radio to infrared SEDs are best-fitted by power law plus graybody models of dust residing in the central galactic regions within a $2.4 \mathrm{kpc}$ diameter and with temperatures between $\sim 21$ and $28 \mathrm{~K}$, emissivity index $\simeq 2$, and masses from $\sim 1.6$ to $19 \times 10^{5} M_{\odot}$. The emissivity index is consistent with dust constituting amorphous silicate and carbonaceous grains previously modeled for stellar-heated dust observed in the Galaxy and other nearby extragalactic sources. Using updated dust absorption coefficients for this type of dust, dust masses are estimated that are similar to those determined from earlier FIR data alone, even though the latter results implied hotter dust temperatures. Fluxes and masses that are consistent with the new FIR and submillimeter data are estimated for dust cooler than $20 \mathrm{~K}$ within the central galactic regions. Tighter physical constraints for such cold, diffuse dust (if it exists) with low surface brightness will need sensitive FIR to submillimeter observations with the Spitzer Space Telescope, SCUBA2, or ALMA.
\end{abstract}

Subject headings: galaxies: elliptical and lenticular, $\mathrm{cD}$ — galaxies: ISM — galaxies: photometry — infrared: galaxies — radio continuum: galaxies — submillimeter

\section{INTRODUCTION}

Optical absorption patches, lanes, and filaments of dust have been seen in $50 \%-80 \%$ of nearby bright elliptical galaxies (e.g., van Dokkum \& Franx 1995). Observations at a range of other wavelengths have also revealed unexpected amounts of gas and dust in these galaxies (e.g., Roberts et al. 1991; Goudfrooij et al. 1994). In some objects, the dust masses estimated from optical extinction studies are a magnitude lower than masses implied by the IRAS far-infrared (FIR) fluxes, suggesting that elliptical galaxies may contain diffusely distributed dust not detected or properly accounted for in optical observations (e.g., Goudfrooij \& de Jong 1995). The dust would add another degeneracy to age-metallicity degeneracies, further complicating the interpretations of broadband colors (e.g., Wise \& Silva 1996). Attenuation by the dust would also produce effects similar to the expected kinematic signature of a dark matter halo and thus provide an alternative explanation for the usual stellar kinematic evidence for dark matter halos around elliptical galaxies (e.g., Baes \& Dejonghe 2002). Therefore, constraining the physical properties of the dust is very crucial.

The IRAS FIR beams were greater than $1^{\prime}$ and sampled only the spectrum of dust at temperatures greater than $25 \mathrm{~K}$,

\footnotetext{
1 Ritter Astrophysical Research Center, University of Toledo, Mail Stop 113, 2801 West Bancroft Street, Toledo, OH 43624; leeuw@astro1.panet .utoledo.edu.

${ }^{2}$ Centre for Astrophysics, University of Central Lancashire, Preston PR1 2HE, UK; aesansom@uclan.ac.uk.

${ }^{3}$ Astronomy Technology Centre, Royal Observatory, Blackford Hill, Edinburgh EH93HJ, UK; eir@roe.ac.uk.

4 Astronomisches Institut, Ruhr-Universität Bochum, 44780 Bochum, Germany; haas@astro.ruhr-uni-bochum.de.

5 Nobeyama Radio Observatory, Minamimaki, Minamisaku, Nagano 3841305, Japan; kuno@nro.nao.ac.jp.
}

providing poor constraints on the dust distribution and emission from dust at less than $25 \mathrm{~K}$. Using the previous generation submillimeter detector UKT14 (Duncan et al. 1990), Knapp \& Patten (1991) did not detect any submillimeter dust emission from their sample of radio galaxies, which included many $I R A S$-detected elliptical galaxies. They assumed dust temperatures of $\sim 18 \mathrm{~K}$, as estimated for Galactic dust (e.g., Reach et al. 1995), and boosted by the lower dust temperatures, they inferred large dust contents of $\sim 10^{6}-10^{8} M_{\odot}$, i.e., as found in luminous spiral galaxies. Millimeter and submillimeter continuum emission was subsequently detected from a sample of early-type galaxies by Fich \& Hodge (1993; also using UKT14). Results from these data showed that dust masses in early-type galaxies were an order of magnitude lower than seen in spirals. In a recent review of cold gas in elliptical galaxies, Knapp (1999) concluded that field elliptical galaxies contain about $0.01-0.1$ of the interstellar matter content of spiral galaxies of similar luminosity. Because of the dust's relative paucity, detecting its total content in elliptical galaxies is much harder.

A reexamination of the $I R A S$ detection rate and fluxes by Bregman et al. (1998) showed that most IRAS detections of early-type galaxies were near the $3 \sigma$ level, with only about $12 \%$ of the galaxies in their sample having been detected above the $98 \%$ confidence level. This showed that although dust might be present in a number of early-type galaxies, a record of good FIR fluxes, which were crucial for constraining dust properties in elliptical galaxies, existed for only a few early-type galaxies. Recent extensive work by Temi et al. (2004) added ISO archival data for 39 giant elliptical galaxies to the FIR data record and is expected to be complemented by new FIR data from the recently launched Spitzer Space Telescope.

This paper extends the previous FIR to millimeter studies of early-type galaxies, exploiting newer, sensitive instruments by 
exploring whether $2 \mathrm{~mm}$ to $450 \mu \mathrm{m}$ data obtained with NOBA and SCUBA (which is 100 times more sensitive than UKT14; Holland et al. 1999) can reveal any dust component at less than $20 \mathrm{~K}$ and constrain the total content in selected nearby field elliptical galaxies. The millimeter to submillimeter measurements from NOBA and SCUBA, which are obtained respectively at the Nobeyama ${ }^{6}$ and James Clerk Maxwell Telescopes $(\mathrm{JCMT}),{ }^{7}$ are used together with archival 60 and $100 \mu \mathrm{m} I R A S-$ $\mathrm{NED}^{8}$ and new $60-200 \mu \mathrm{m} I S O$ data, ${ }^{9}$ with the aim of tightening physical properties of cold dust that emits in these wave bands. The thermal emission fluxes from the millimeter to FIR continuum are analyzed together with some archival radio data obtained from NED to account for any nonthermal, highfrequency radio synchrotron contribution to the cold dust spectrum. Some IRAS data were reprocessed using HIRES and SCANPI routines at the NASA/IPAC Center and used to check the data for any FIR source confusion or contamination.

\section{SOURCE SELECTION, FAR-INFRARED DATA, AND SUBMILLIMETER TO MILLIMETER OBSERVATIONS}

The elliptical galaxies selected for submillimeter to millimeter observations had IRAS-detected 60 and $100 \mu \mathrm{m}$ emission that was thought to indicate excess dust over their optically determined dust masses (e.g., Goudfrooij et al. 1994; Wise \& Silva 1996). In order to maximize the detection of expected low-level fluxes and with the aim of spatially resolving them, the targets are relatively strong FIR emitters $(>100 \mathrm{mJy})$ and nearby $(<45 \mathrm{Mpc})$. To avoid confusing any nonthermal flux contribution to the detected thermal emission the galaxies, apart from NGC 4374, are low radio emitters. They include elliptical galaxies that had inferred dust masses greater than $\sim 10^{5} M_{\odot}$ in samples by Knapp \& Patten (1991), Goudfrooij \& de Jong (1995), and Bregman et al. (1998).

ISOPHOT photometry observations from 60 to $200 \mu \mathrm{m}$ (Lemke et al. 1996) of the five galaxies NGC 3962, NGC 4374, NGC 4697, and NGC 5353/NGC 5354 were obtained from the ISO Data Archive (Kessler et al. 2000). They were reduced using the PHOT Interactive Analysis tool (PIA, ver. 9.1) together with the calibration data set, version 7.0, providing a photometric accuracy of better than $30 \%$ for faint sources (Laureijs \& Klaas 1999).

NGC 3962, NGC 4374, and NGC 4697 were observed in chopped mode (ISOPHOT AOT 22) and NGC 5353/5354 in sparse mapping mode (ISOPHOT AOT 37/39). The data reduction included correction for the electronics' nonlinearity, removal of data sections contaminated by cosmic particle events (also known as deglitching), and correction for signal dependence on the reset interval time. For the chopped observations, the data reduction also included correction for transient effects using the so-called pattern analysis method.

\footnotetext{
${ }^{6}$ The Nobeyama Telescope is operated by the Nobeyama Radio Observatory (NRO), which is a branch of the National Astronomical Observatory of Japan.

7 The JCMT is operated by the Joint Astronomy Centre on behalf of the United Kingdom Particle Physics and Astronomy Research Council (PPARC), the Netherlands Organisation for Scientific Research, and the National Research Council of Canada.

${ }^{8}$ The NASA/IPAC Extragalactic Database (NED) is operated by the Jet Propulsion Laboratory, California Institute of Technology, under contract to the National Aeronautics and Space Administration.

9 Based on observations with the Infrared Space Observatory (ISO), an ESA project with instruments funded by ESA Member States (especially the PI countries: France, Germany, the Netherlands, and the United Kingdom), and with the participation of ISAS and NASA.
}

As a cross-check on this technique, a Fourier analysis method was also applied as described in detail by Haas et al. (2000), yielding similar results to within $10 \%$. Further reduction included the calibration of the detector responsivity and its changes, performed using associated measurements of the thermal fine calibration source on board.

All ISO source fluxes were corrected for aperture size assuming an unresolved point source, since on the ISOPHOT C100 array (which has $3 \times 3$ pixels of diameters $46^{\prime \prime}$ ) no indication of any significant, extended $60-100 \mu \mathrm{m}$ flux was found beyond the pixel resolution. The photometry in the $60-$ $100 \mu \mathrm{m}$ wave bands was derived from the $46^{\prime \prime}$ central C100 pixel pointed at the source. For the chopped 170 and $200 \mu \mathrm{m}$ observations, where the sources were centered on the $2 \times$ 2 pixel array, the photometry was derived from the whole array $\left(180^{\prime \prime}\right)$, as in that case the source flux was measured by all 4 pixels (see, e.g., Fig. 1, top left panel). Slightly different flux extraction was required for the $180 \mu \mathrm{m}$ observations of the companion galaxies NGC 5353 and NGC 5354, because both objects were detected by this array simultaneously (see details below).

The top panels in Figure 1 show that the galaxies NGC 5353 and NGC 5354 are only about $70^{\prime \prime}$ apart, with the latter being in the north. This is sufficiently close for the two galaxies to be covered by the ISOPHOT C100 and C200 arrays, which have $3 \times 3$ and $2 \times 2$ pixels of size $46^{\prime \prime}$ and $92^{\prime \prime}$ each, respectively, and to allow the galaxies to be resolved on the C100 array. As shown in the left panels in Figure 1, NGC 5353 and NGC 5354 were respectively observed exactly with the center and corner pixels of the C100 array, ensuring that the galaxies would be clearly resolved from each other on this array. Those C100 pixels that are not centered on the two sources show some low-level flux (Fig. 1, bottom left histogram), which stems from the wings of the point-spread function, primarily of the central source. The same applies for the observations at $60 \mu \mathrm{m}$. The right panels of Figure 1 show that at $180 \mu \mathrm{m}$ NGC 5353 is centered on the $2 \times 2$ pixels of the C200 array, so that its flux is distributed over all 4 pixels, and NGC 5354 is located on the corner pixel containing most of its flux. In order to separately extract the $180 \mu \mathrm{m}$ fluxes of the two galaxies on the C200 array, two point sources were fitted to the measured fluxes: one was centered on the array, and the other located almost exactly on a corner pixel, corresponding to the C100 array positions of NGC 5353 and NGC 5354, respectively. This paper exploits the new ISO data of NGC 5354 and NGC 5353 and deals with these companion galaxies as individual FIR sources. For the first time ever (see details about the corresponding IRAS measurements below), the two galaxies are resolved from each other at 60 and $100 \mu \mathrm{m}$, and their fluxes are separately extracted at $180 \mu \mathrm{m}$. It is noted that Temi et al. (2004) did not resolve the pair NGC 5354 and NGC 5353 in their recent, extensive analysis of the same ISO data as presented here.

Photometric observations in the submillimeter were obtained with SCUBA on various nights from 2001 January to 2002 February. The camera was simultaneously operated with the central bolometers of the shortwave array at $450 \mu \mathrm{m}$ and the longwave array at $850 \mu \mathrm{m}$. The photometry employed a nine-point jiggle pattern in a $3 \times 3$ grid of $2^{\prime \prime}$, and standard SCUBA data reduction was undertaken (e.g., Leeuw et al. 2000). The fluxes were calibrated using instrumental gains determined from photometry observations of planets and, when planets were not available, the JCMT secondary calibrators (Sandell et al. 1998; Jenness et al. 2002). 

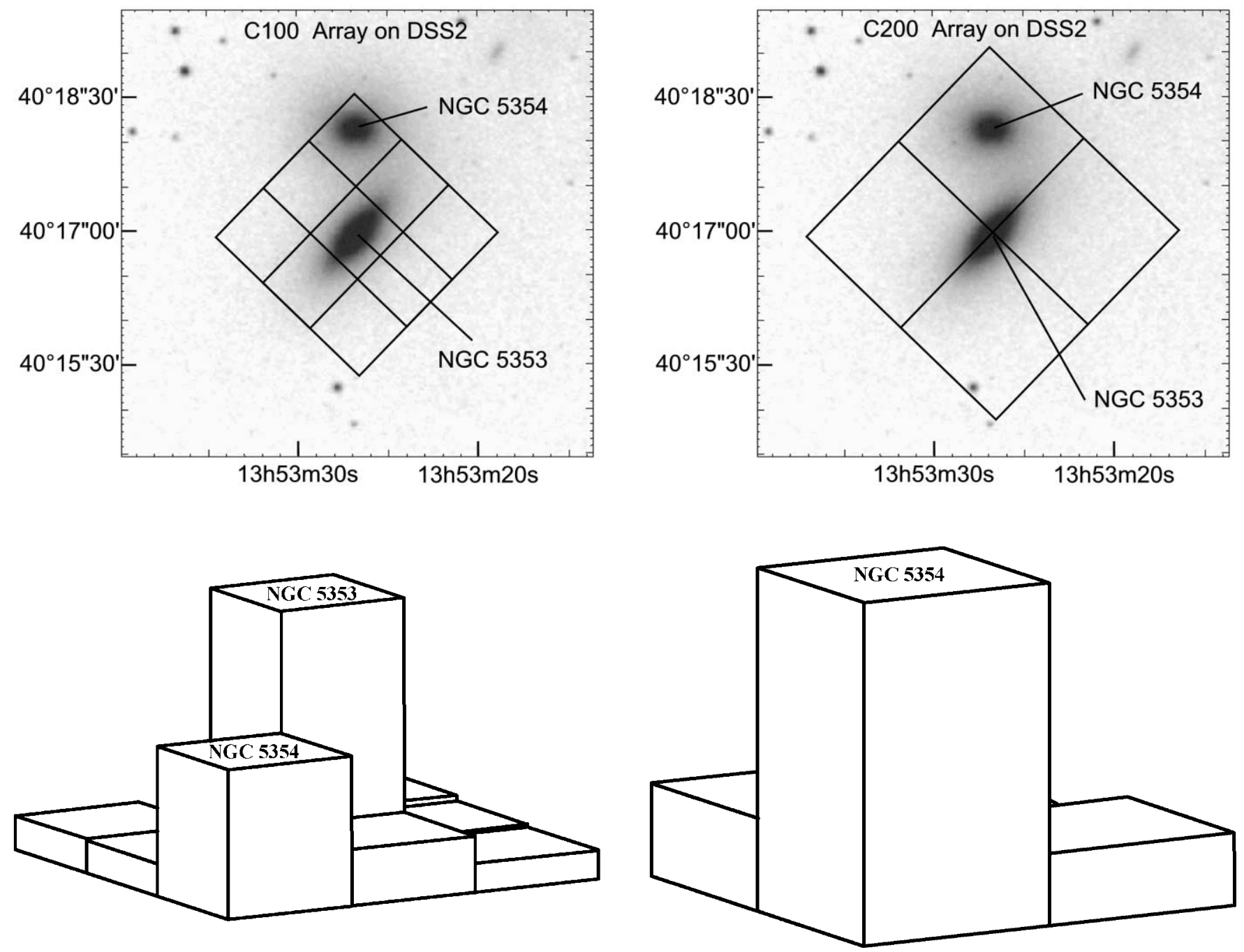

FIG. 1.- Infrared Digitized Sky Survey 2 images of NGC 5353 and NGC 5354 with superposed ISOPHOT C100 (top left) and C200 (top right) array pointings that were used for the ISO observations of these galaxies. Bottom left and right: Three-dimensional histograms of the ISO 100 and $180 \mu \mathrm{m}$ fluxes, respectively, before the individual galaxy fluxes were corrected for aperture size (see text). For visual clarity, the histograms are oriented so that the fainter source is plotted in the foreground.

On 2002 March 4, the elliptical galaxies NGC 4374, NGC 4697, and NGC 5353 were scan-mapped at $2 \mathrm{~mm}$ with the NOBA bolometer array attached to the Nobeyama $45 \mathrm{~m}$ Telescope. During the telescope allocation for this study, only three objects were observed because there was only a limited spell of good weather. The typical sky opacity $(\tau)$ during the observation was 0.12 , measured using skydips. Exposure times were $\sim 90$ minutes per galaxy, raster scanning across each target to obtain maps of $1^{\prime} \times 1^{\prime}$. Primary flux calibration observations were obtained on Saturn, and secondary calibration on 3C 273 and 3C 279. The expected flux for Saturn was obtained from the JCMT planetary flux program, and those for 3C 273 and 3C 279 were bootstrapped from JCMT monitoring observations. There was no indication of extended emission beyond the NOBA 12" beam (FWHM) at $2 \mathrm{~mm}$. The resulting FIR to millimeter flux estimates and upper limits are presented in Table 1.

There is a discrepancy between the IRAS and ISO fluxes of NGC 5353 and NGC 5354; this is because on IRAS scans NGC 5354 was not resolved from NGC 5353, and only the combined flux of the galaxy pair was listed in the IRAS Point
Source Catalog (PSC). Compared with the combined ISO fluxes of the galaxies, the IRAS PSC flux is much smaller, i.e., only about $50 \%$. The baseline determined by the IRAS PSC algorithm generally uses an unresolved point-source filter, so that for a moderately extended source like the pair NGC 5353/5354 the baseline is set at the level of the extended emission, i.e., too high, resulting in too low a source flux. A similar trend of too-low IRAS PSC fluxes was found for 12 moderately resolved spiral galaxies (Bendo et al. 2002; see their $\S 4.1 .3$ ). An examination of the SCANPI-processed IRAS scans around NGC 5353 shows that the low IRAS fluxes might be caused by a poor baseline determination on the pair NGC $5353 / 5354$, plus some extended flux contribution from another $4^{\prime}$ nearby source. A visual inspection of the scans and baselines for the pair gives $F(60 \mu \mathrm{m})=0.45 \mathrm{Jy}$ and $F(100 \mu \mathrm{m})=1.9 \mathrm{Jy}$, which comes close to the $I S O$ values of $F(60 \mu \mathrm{m})=0.6 \mathrm{Jy}$ and $F(90 \mu \mathrm{m})=1.95 \mathrm{Jy}$. This leads to a conclusion that the 60 and $100 \mu \mathrm{m} I S O$ fluxes of NGC 5353 and NGC 5354 in this paper are correct and that the newly determined fluxes are used in the spectral energy distribution (SED) analyses below. It is interesting that despite the decomposition of the $180 \mu$ m fluxes, the 
TABLE 1

Far-Infrared to Millimeter Fluxes of Nearby IRAS Bright Elliptical Galaxies

\begin{tabular}{|c|c|c|c|c|c|c|c|c|c|}
\hline \multirow[b]{2}{*}{ GALAXY NaME } & \multicolumn{2}{|c|}{$I R A S$} & \multicolumn{4}{|c|}{ ISO } & \multicolumn{2}{|c|}{ SCUBA } & \multirow[b]{2}{*}{ NOBA $(2 \mathrm{~mm})$} \\
\hline & $(60 \mu \mathrm{m})$ & $(100 \mu \mathrm{m})$ & $(60 \mu \mathrm{m})$ & $(90 \mu \mathrm{m})$ & $(170 \mu \mathrm{m})$ & $(200 \mu \mathrm{m})$ & $(450 \mu \mathrm{m})$ & $(850 \mu \mathrm{m})$ & \\
\hline NGC $2986 \ldots \ldots \ldots . .$. & $<0.05$ & $0.36 \pm 0.11$ & $\ldots$ & $\ldots$ & $\ldots$ & $\ldots$ & $<0.13$ & $<0.010$ & $\ldots$ \\
\hline NGC $3156 \ldots \ldots \ldots . .$. & $0.22 \pm 0.04$ & $0.91 \pm 0.09$ & $\ldots$ & $\ldots$ & $\ldots$ & $\ldots$ & $<0.06$ & $0.006 \pm 0.002$ & $\ldots$ \\
\hline NGC $3962 \ldots \ldots \ldots . .$. & $0.21 \pm 0.04$ & $<0.98$ & $0.30 \pm 0.06$ & $0.76 \pm 0.10$ & $0.81 \pm 0.15$ & $<0.91$ & $<0.12$ & $<0.009$ & $\ldots$ \\
\hline NGC $4374 \ldots \ldots \ldots . .$. & $0.50 \pm 0.06$ & $0.98 \pm 0.21$ & $0.70 \pm 0.10$ & $1.00 \pm 0.10$ & $0.70 \pm 0.20$ & $<0.90$ & $0.12 \pm 0.02$ & $0.147 \pm 0.020$ & $0.129 \pm 0.010$ \\
\hline NGC $4697 \ldots \ldots \ldots . .$. & $0.59 \pm 0.05$ & $1.24 \pm 0.08$ & $1.15 \pm 0.35^{\mathrm{a}}$ & $1.45 \pm 0.35^{\mathrm{a}}$ & $0.85 \pm 0.25$ & $0.75 \pm 0.20$ & $\ldots$ & $<0.007$ & $<0.010$ \\
\hline NGC $5353 \ldots \ldots \ldots .$. & $0.32 \pm 0.06$ & $1.45 \pm 0.11$ & $0.40 \pm 0.10$ & $1.25 \pm 0.10^{\mathrm{b}}$ & $0.60 \pm 0.20^{\mathrm{c}}$ & $\ldots$ & $\ldots$ & $0.017 \pm 0.002$ & $0.011 \pm 0.003$ \\
\hline NGC $5354 \ldots \ldots \ldots . .$. & $0.41 \pm 0.05$ & $1.61 \pm 0.06$ & $0.20 \pm 0.06$ & $0.70 \pm 0.10^{\mathrm{b}}$ & $0.58 \pm 0.20^{\mathrm{c}}$ & $\ldots$ & $\ldots$ & $\ldots$ & $\ldots$ \\
\hline
\end{tabular}

Note.-All listed fluxes are in units of Jy, and errors and upper limits are 1 and $3 \sigma$, respectively.

a There is a large uncertainty in the onboard calibration measurement for this flux.

b This is an ISO $100 \mu \mathrm{m}$ filter measurement from the C100 array.

c This is an ISO $180 \mu \mathrm{m}$ filter measurement from the C200 array.

SEDs of NGC 5353 and NGC 5354 are not much different from those of the other sources presented in this paper (see $\S 4$ ).

\section{BEAM SIZES AND SOURCE FLUXES AND EXTENTS}

The fluxes presented here (see Table 1) come from singlepointing observations obtained with beams that vary in size. The IRAS 60 and $100 \mu \mathrm{m}$ fluxes are $\sim 120^{\prime \prime}-240^{\prime \prime}$, ISO 170 $200 \mu \mathrm{m} \mathrm{180"} \mathrm{(except} 92^{\prime \prime}$ for NGC 5353 and NGC 5354), ISO $60-100 \mu \mathrm{m} \sim 46^{\prime \prime}$, SCUBA $850 \mu \mathrm{m} \sim 16^{\prime \prime}$, NOBA $2 \mathrm{~mm} \sim 12^{\prime \prime}$, and SCUBA $450 \mu \mathrm{m} \sim 9^{\prime \prime}$ (FWHM in all cases, except the $180^{\prime \prime}$ for the ISO $170-200 \mu \mathrm{m}$ flux is $2 \times \mathrm{FWHM}$ ). Therefore, if the FIR to submillimeter emission from the targets is as extended as the galaxies' effective radii, i.e., $14^{\prime \prime}-72^{\prime \prime}$ (see Table 2), then for each object the fluxes from the respective instruments will come from different galactic regions corresponding to the sampling beam sizes.

Mindful of this, and for reasons noted below, the SED analyses here assume that all the fluxes come from predominantly unresolved, central regions of the galaxies, whose extent is less than the employed millimeter to $I S O 100 \mu \mathrm{m}$ beams, i.e., less than $8^{\prime \prime}$ to less than $46^{\prime \prime}$, or roughly less than $1 \mathrm{kpc}$ to less than $6 \mathrm{kpc}$ galactic scales. As noted earlier, at least $50 \%$ of the nearby bright elliptical galaxies have dust features that manifest absorbing optical light within areas of such nuclear regions. Some elliptical galaxies also show a compact nucleus associated with radio emission in some of these galaxies (e.g., Bower et al. 1997). The submillimeter emission from these cores is expected to be the high-frequency extrapolation of synchrotron radiation that is responsible for the radio source. Leeuw et al. (2002) showed that this emission can be comparable to the total submillimeter flux from low-level dust emission and therefore needs to be accounted for when analyzing any possible submillimeter reradiation from dust, especially in unresolved sources with active nuclei.

The assumption that the FIR to submillimeter emission comes from a dominant unresolved region in the presented observations is made following NOBA-, SCUBA-, and ISOPHOTmapping results of three of the galaxies studied here, i.e., NGC 4374, NGC 5353, and NGC 5354. First, the SCUBA mapping observations by Leeuw et al. (2000) showed that the $850 \mu \mathrm{m}$ continuum from this object was unresolved, constraining the emitting source by the SCUBA 850 resolution to less than $16^{\prime \prime}$. Second, the NOBA mapping results of NGC 4374 and NGC 5353 constrain the $2 \mathrm{~mm}$ emission from these galaxies by the NOBA resolution to less than $12^{\prime \prime}$.
Similarly, the ISOPHOT mapping results constrain the $100 \mu \mathrm{m}$ emission from NGC 5353 and NGC 5354 to less than $46^{\prime \prime}$. Consistent with the above assumption, the parameter results of the SED model fitting require that the emitting graybody sources in all the studied elliptical galaxies are less than about $9^{\prime \prime}$ (see below). This is true for single-temperature graybody sources with the standard dust emissivity indices from 1 to 2 . However, when the SED model fitting is computed for two(or more) temperature graybody sources (Leeuw et al. 2002), where one of the temperatures is considerably lower than the results presented in this paper, larger emitting regions for the coldest dust could be implied.

Removing the assumption that the emitting regions detected here are unresolved could have some consequence on the fitting and derived parameters. In particular, if the detected FIR to millimeter emission is extended, the larger FIR beams could include emission from outside the very central regions $\left(\sim 8^{\prime \prime}-\right.$ $16^{\prime \prime}$ ) sampled by the millimeter and submillimeter beams and imply that the millimeter and submillimeter fluxes are undersampled relative to the FIR data in the SED fits. The SED fitting to the "undersampled," lower submillimeter fluxes $\left(F_{\nu}\right.$ or $S_{\nu}$ below) would (1) require higher emissivity index $(\beta)$ values to match the slope at the Rayleigh-Jeans tail, (2) imply smaller emitting region or scaling factor $(\Omega)$ values (from eq. [1]; $F_{\nu} \propto \Omega$ ), and (3) yield lower dust mass determinations (from eq. [3]; $M_{d} \propto S_{\nu}$ ) than would be estimated with the higher submillimeter fluxes.

\section{DECOMPOSING THE SED}

To model the radio to infrared SED, a combination of a power law (nonthermal radio emission from the galaxy's nucleus) plus graybody (reprocessed emission from dust) was used. The composite model flux is

$$
F_{\nu}=C \nu^{\alpha}+\Omega B_{\nu}(T)\left\{1-\exp \left[-\left(\frac{\lambda_{0}}{\lambda}\right)^{\beta}\right]\right\},
$$

where $C$ is the normalization for the power-law component, $\alpha$ is the power-law spectral slope with $F_{\nu} \propto \nu^{\alpha}, \Omega$ is the solid angle for the graybody component, $B_{\nu}(T)$ is the Planck function at temperature $T, \lambda_{0}$ is the wavelength at which the optical depth is unity, and $\beta$ is the emissivity index of the grains. Because of the limited frequency sampling in the data, the temperature $T$ and its uncertainty are the only key parameters 
TABLE 2

Power Law Plus Graybody Fitting Results for Nearby iRas Bright Elliptical Galaxies

\begin{tabular}{|c|c|c|c|c|c|c|c|c|c|c|c|c|}
\hline Galaxy Name & Galaxy Type & $\begin{array}{l}\text { Effective Radii } \\
\quad(\operatorname{arcsec})\end{array}$ & $\begin{array}{c}D \\
(\mathrm{Mpc})\end{array}$ & Power-Law ${ }^{\mathrm{a}}$ Index & $\begin{array}{l}T_{\text {dust }} \\
(\mathrm{K})\end{array}$ & $\begin{array}{l}\Omega_{\text {dust }} \\
(\mathrm{sr})\end{array}$ & Reduced $\chi^{2}$ & $\begin{array}{c}M_{\mathrm{dust}}^{60 \mu \mathrm{m}} \\
\left(\log M_{\odot}\right)\end{array}$ & $\begin{array}{c}T_{\text {dust }}^{\mathrm{b}} \\
(\mathrm{K})\end{array}$ & $\begin{array}{c}\text { Reduced }^{\mathrm{b}} \\
\chi^{2}\end{array}$ & $\begin{array}{c}T_{\text {dust }}^{\mathrm{c}} \\
(\mathrm{K})\end{array}$ & $\begin{array}{c}\text { Reduced }^{\mathrm{c}} \\
\chi^{2}\end{array}$ \\
\hline NGC $2986 \ldots \ldots \ldots \ldots . . .$. & E2 & 36 & 40.2 & -0.50 & $\sim 21(<32)$ & $(1.04 \mathrm{E}-9)^{\mathrm{d}}$ & N/A & $(\sim 6.28)^{\mathrm{d}}$ & $\sim 23$ & N/A & N/A & $\mathrm{N} / \mathrm{A}$ \\
\hline NGC $3156 \ldots \ldots \ldots \ldots$ & $\mathrm{E} / \mathrm{SO}$ & 14 & 22.3 & -0.25 & $24.6 \pm 0.9$ & $1.26 \mathrm{E}-9$ & 0.1 & $5.60 \pm 0.02$ & 28.7 & 3.8 & N/A & N/A \\
\hline NGC $3962 \ldots \ldots \ldots \ldots . . . .$. & E1 & 35 & 32.4 & -0.25 & $24.0 \pm 0.7$ & $1.67 \mathrm{E}-9$ & 1.3 & $5.67 \pm 0.02$ & 26.2 & 1.2 & $12 / 24$ & 2.0 \\
\hline NGC $4374 \ldots \ldots \ldots \ldots . . .$. & E1 & 51 & 20.7 & -0.25 & $28.1 \pm 0.9$ & $0.84 \mathrm{E}-9$ & 2.5 & $5.20 \pm 0.02$ & 31.5 & 2.8 & $12 / 31$ & 4.6 \\
\hline NGC $4697 \ldots \ldots \ldots \ldots . . . .$. & E6 & 72 & 21.4 & -0.25 & $28.1 \pm 0.7$ & $0.94 \mathrm{E}-9$ & 1.4 & $5.60 \pm 0.02$ & 31.2 & 0.8 & $12 / 28$ & 1.6 \\
\hline NGC 5353............... & $\mathrm{E} / \mathrm{SO}$ & 15 & 45.1 & -0.30 & $25.3 \pm 0.9$ & $1.46 \mathrm{E}-9$ & 1.0 & $6.15 \pm 0.02$ & 29.2 & 2.5 & $9 / 27$ & 2.2 \\
\hline NGC 5354............. & SAO: sp & 18 & 45.1 & -0.25 & $24.6 \pm 1.8$ & $9.00 \mathrm{E}-10$ & 0.1 & $6.16 \pm 0.04$ & 26.9 & 0.01 & N/A & N/A \\
\hline
\end{tabular}

Notes.-The galaxy types and effective radii were respectively obtained from NED and de Vaucouleurs et al. (1991). The distances listed in the fourth column are from earlier FIR studies Roberts et al. 1991; Goudfrooij \& de Jong 1995) to best facilitate comparison. The listed uncertainties are $1 \sigma$.

a The adopted power-law slope of $-0.25 \pm 0.3$ for NGC 4374 is that previously fitted to the radio to submillimeter data by Leeuw et al. (2000). As only limited radio to submillimeter data are available for the other objects, the other indices are fixed as close as the fitting reasonably permits to the value for NGC 4374

${ }^{\mathrm{b}}$ These values are for $\beta=1.5$, for comparison to the values for $\beta=2$ adopted in this paper. For NGC 3962 and NGC 4697 , which have better $\beta=1.5 \chi^{2}$ values than $\beta=2 \chi^{2}$ values, submillimeter upper limits not included in the $\chi^{2}$ determination fall below the $\beta=1.5$ model plots and favor the $\beta=2$ model.

c These values are for two-temperature components with $\beta=2.0$, for comparison to the values for single-temperature model fits.

d The parentheses indicate a large uncertainty due to limited data, and the dust mass is calculated from the $100 \mu \mathrm{m}$ flux. 
that are statistically determined in equation (1). Therefore, in fitting equation (1), the factors $C$ and $\Omega$ were normalized to the data, the temperature was allowed to vary and statistically computed, and the power-law index $\alpha$ and emission parameters $\lambda_{0}$ and $\beta$ were fixed and adopted, as described below.

The normalizing factors $C$ and $\Omega$ were obtained by forcing the model flux to agree with the core radio $(1.4$ and $5 \mathrm{GHz})$ and FIR (60 and $100 \mu \mathrm{m}$ ) fluxes, respectively, since the two components dominate in these different wave bands, and the bestfitting values for the temperatures and their uncertainties were determined by minimizing $\chi^{2}$ for preset values of $\beta=1$, $1.5,1.8$, and 2 in equation (1). Following Leeuw et al. (2000) and references therein, $\lambda_{0}=7.9 \mu \mathrm{m}$ and $\alpha=-0.25$ were fixed and used on fits for all objects but NGC 2986 and NGC 5353, for which, from examinations of their fits by eye, the millimeter and submillimeter data clearly required steeper powerlaw slopes of $\alpha=-0.5$ and -0.3 , respectively (see Table 2).

The lowest $\chi^{2}$ values, i.e., best fits, were obtained with $\beta=2$, and the worst fits were obtained with $\beta=1$. In general, the fits became worse and yielded slightly higher temperatures with decreasing $\beta$. Relative to values obtained with $\beta=2$, the temperatures obtained with $\beta=1.8$ and 1.5 were greater by $T \sim 2$ and $\sim 4 \mathrm{~K}$, respectively. Table 2 lists the temperatures and $\chi^{2}$ values for $\beta=1.5$, as a demonstration of the higher temperatures and poorer fits with $\beta<2$. The table also shows that while values of $\beta=2$ look best, there is not much choice between that and values of $\beta=1.5$, indicating the degree of uncertainty in the fits. For temperature values determined here, a choice of $\beta$ primarily affects the SEDs longward of $100 \mu \mathrm{m}$, so that for NGC 3962 and NGC 5354, for which there are only flux upper limits or no FIR to submillimeter data points longward of $100 \mu \mathrm{m}$, the $\beta$ providing the best fit (i.e., lowest $\chi^{2}$ ) for these objects cannot be discriminated. For NGC 4374, the differences in the 60 and $100 \mu \mathrm{m}$ fluxes are the dominant contributor to the $\chi^{2}$; therefore, the $\chi^{2}$ with different $\beta$ values for this object are about the same.

Mindful of the fitting uncertainties, the general deduction in this paper is that the reduced $\chi^{2}$ values that are listed in the Table 2 analysis demonstrate that single-temperature graybodies with $\beta=2$ produced reasonable SED fits for the objects presented here (see also $\S 4.2$ ). For NGC 3962 and NGC 4697, which appear to have better $\beta=1.5 \chi^{2}$ values than $\beta=2 \chi^{2}$ values, submillimeter upper limits not included in the $\chi^{2}$ determination fall below the $\beta=1.5$ model plots but are consistent with the $\beta=2$ plots. Therefore, graybodies with temperatures obtained from these $\beta=2$ fits are adopted in this paper. They are plotted as dotted lines in Figure 2, and their derived parameters are listed in Table 2. The power law plus graybody model is plotted as solid or dashed lines (see Fig. 2), respectively, for power-law indices of NGC 4374, which were determined from linear regression fits by Leeuw et al. (2000), and of the other galaxies, which have been fixed following the results of NGC 4374 as described above. New millimeter to submillimeter observations obtained with the Nobeyama Telescope and SCUBA are marked by open squares and circles, respectively. New FIR data from ISO are indicated by asterisks. Other infrared 100 and $60 \mu \mathrm{m}$ data in the figures are from IRAS, which together with all radio data were obtained from NED. The downward arrows indicate $3 \sigma$ upper limits.

\subsection{Limitations of Data and SEDs for Individual Galaxies}

Because of the submillimeter and millimeter faintness of sources presented and the limited sensitivity of instruments used, about half of the submillimeter and millimeter data points are upper limits. Therefore, some galaxies in the sample have little or incomplete millimeter to FIR observational data sets to allow a model fitting to the SEDs with the full range of variables that could be considered free parameters in equation (1). Also, some ISO data duplicate the IRAS measurements at 60 and $100 \mu \mathrm{m}$ such that even when the number of free parameters to fit to the data are restricted as described above (see $\S 4$ ), a few of the galaxies are still left with too few data and too many free parameters in the fitting process.

Specifically, NGC 2986 has only two photometric data points and three upper limits in the millimeter to FIR regime. Therefore, no reduced $\chi^{2}$ was computed for this object. The dust temperature obtained can be thought of as derived from the 60-100 $\mu \mathrm{m}$ flux ratio, as commonly derived for objects with only IRAS data (e.g., Goudfrooij \& de Jong 1995). A tentative composite plot for this object is displayed in Figure 2 to demonstrate the consistency of the millimeter to submillimeter upper limits and FIR data with the model fit. Similarly, a tentative dust mass for this object is computed as described for the other objects below and listed in Table 2.

It is noted that NGC 3156 has a limited number of data points. However, the three points this galaxy has in the FIR to submillimeter region are sufficient for making the required fit to the temperature and normalization of its thermal spectrum. It is also noted that NGC 4697 has only three upper limits in the centimeter to submillimeter region. However, this object has a very good sampling of points around the peak of the thermal spectrum; thus, for NGC 4697, the upper limits in the millimeter to submillimeter region are not needed or used to provide any constraints and are plotted primarily to show that these data are consistent with the fit.

Currently, only limited data are available for fitting the power-law spectrum in the region from 1.4 to $5 \mathrm{GHz}$. As noted earlier, the power-law index is therefore fixed at -0.25 for all the galaxies except NGC 2986 and NGC 5353, where it is estimated as -0.50 and -0.30 , respectively. Fixing the index follows fits of NGC 4374 in earlier work (Leeuw et al. 2000), since this source has the needed points from 1.4 to $5 \mathrm{GHz}$. Commissioning of high-frequency radio instruments such as the Green Bank Telescope should allow data in these wave bands to be obtained for other elliptical galaxies in the future, enabling more reliable correction of nonthermal contribution to the millimeter to FIR thermal flux. As noted earlier, the $C$ parameter normalizes the power-law component of the model to the 1.4 and $5 \mathrm{GHz}$ data, for either detected points or upper limits, depending on the presently scant availability of needed data in literature and archives. This normalization is done even though it is less reliable in cases such as for NGC 3156 and NGC 4697, where only $5 \mathrm{GHz}$ upper limits are currently available.

\subsection{A Second Dust Component with $T<20 \mathrm{~K}$ ?}

SED analyses by Dunne \& Eales (2001) and Klaas et al. (2001), among others, have demonstrated evidence for thermal emission with at least two temperature components and an emissivity index of 2. This was for samples of bright IRAS and ultraluminous infrared galaxies and was deduced not only from rigorous fitting results but also physical considerations. The presence of very cold dust has also been suggested in some individual sources, e.g., the peculiar dwarf elliptical galaxy NGC 205 (Haas 1998) and the merger remnant elliptical galaxy NGC 5128 (Leeuw et al. 2002). 

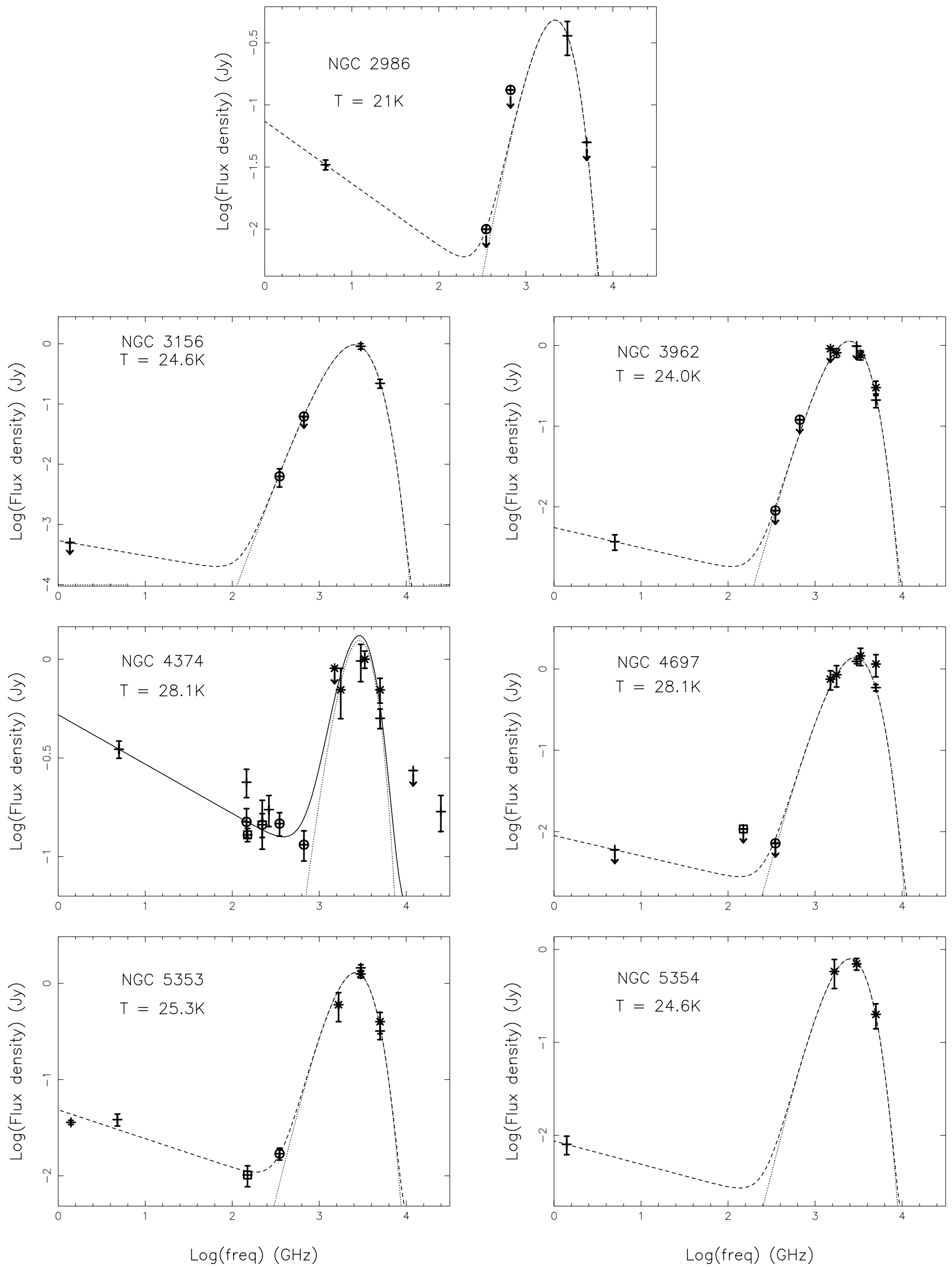

FIg. 2.- Radio to FIR SEDs of central regions in sample FIR-bright elliptical galaxies. The model fits include a composite power law plus graybody given by eq. (1), and together with the data symbols are as described in the text. 

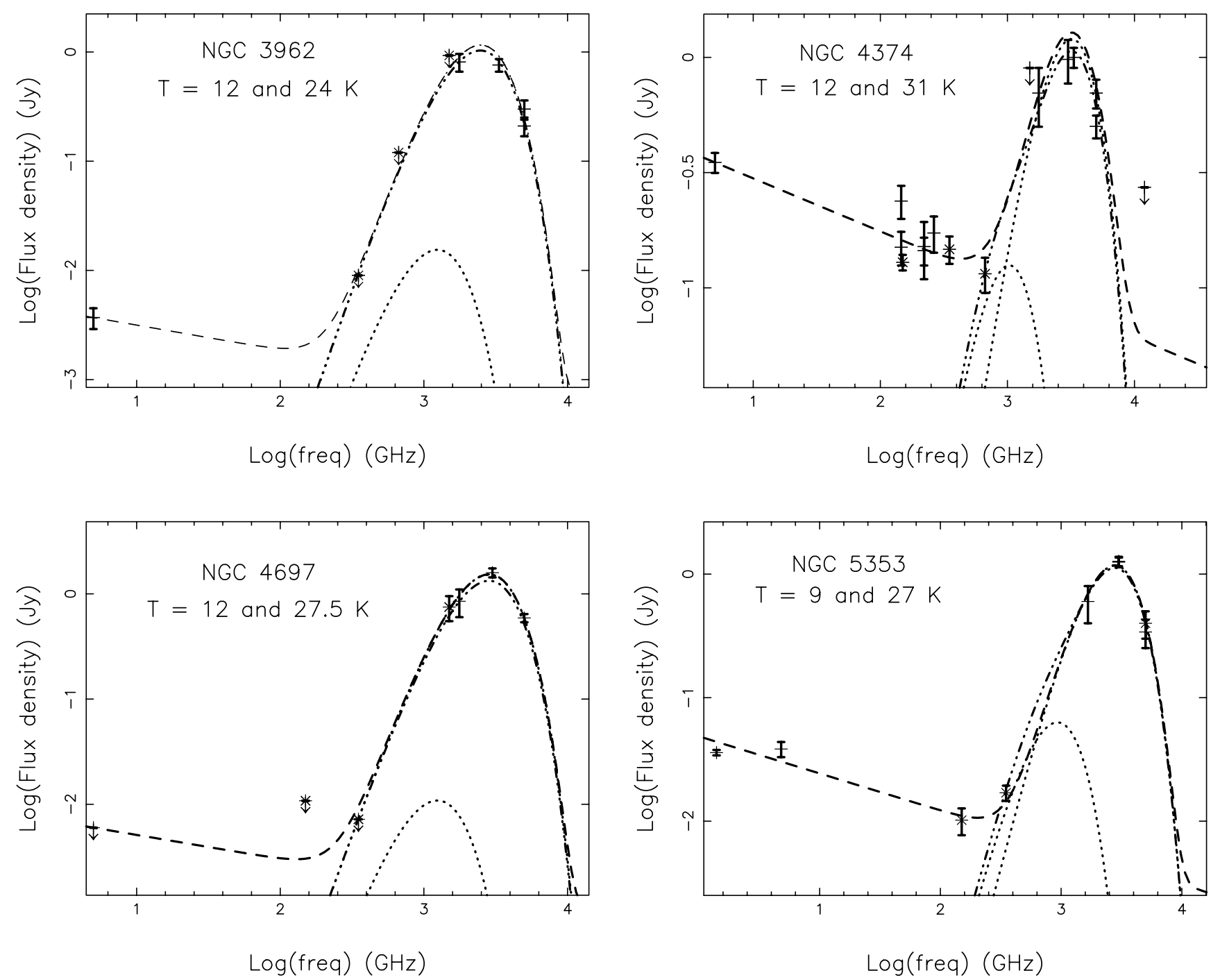

FIG. 3.-Radio to FIR SEDs of central regions in sample FIR-bright elliptical galaxies. The model fits are of a two-temperature graybody given by eq. (2), and together with the data symbols are as described in the text.

Following the investigations by Dunne \& Eales (2001) and Klaas et al. (2001), the FIR to submillimeter data are also fitted with two-component graybodies to test whether such graybodies, including those with components of temperatures less than $20 \mathrm{~K}$ (see Fig. 3), produce better fits than singletemperature models. That the single-temperature graybodies alone do not fit both FIR and submillimeter data simultaneously may indicate that there is a second very cold (i.e., $<20 \mathrm{~K})$ dust component required. The data longward of $100 \mu \mathrm{m}$, as newly available for this paper, can sample fluxes from dust with temperatures not only in the IRAS range of $T \sim 20-100 \mathrm{~K}$ but also at less than $20 \mathrm{~K}$. However, the current data coverage is too sparse in wavelength space, and in some cases the detections have relatively large uncertainties, or only flux upper limits are available. Therefore, it is not possible to reliably determine the exact decompositions of the $6-20 \mathrm{~K}$ (very cold) and 20-40 K (cold) components or securely discriminate whether two-temperature fits are better than singletemperature ones for all galaxies.

For now, the SEDs for which there were at least three data points from 100 to $850 \mu \mathrm{m}$ allow an eyeball fit that is useful for estimating the flux upper limit for the very cold dust temperature component that is consistent with the data. In this regard and to facilitate comparison with fits from equation (1), a two-temperature component graybody of the form

$F_{\nu}=C \nu^{\alpha}+\Omega\left[B_{\nu}\left(T_{1}\right)+B_{\nu}\left(T_{2}\right)\right]\left\{1-\exp \left[-\left(\frac{\lambda_{0}}{\lambda}\right)^{\beta}\right]\right\}$,

where the parameters are as in equation (1), was fitted to the FIR to submillimeter data of NGC 3962, NGC 4374, NGC 4697, and NGC 5353 (see Fig. 3). The modeled flux sums of twotemperature graybodies are normalized to detected FIR or submillimeter fluxes, and it is assumed that the graybodies for respective galaxies have the same solid angles and emissivity indices as obtained from equation (1) and listed in Table 2.

Figure 3 exhibits the composite and individual graybodies that constitute the composites by triple-dot-dashed and purely dotted lines, respectively. Because it is the sum of the two components that is normalized to the data, curves of the composite and individual graybodies respectively fall on and below the submillimeter data. For NGC 3962, the observed $850 \mu \mathrm{m}, 3 \sigma$ limit of $0.009 \mathrm{Jy}$ is fitted by 12 and $24 \mathrm{~K}$ graybodies with modeled $850 \mu \mathrm{m}$ fluxes of 0.0012 and $0.0078 \mathrm{Jy}$, respectively; for NGC 4374 , the observed $450 \mu \mathrm{m}$ 
flux of $0.110 \mathrm{Jy}$ is fitted by 12 and $31 \mathrm{~K}$ graybodies with modeled $450 \mu \mathrm{m}$ fluxes of 0.086 and $0.034 \mathrm{Jy}$, respectively; for NGC 4697, the observed $850 \mu \mathrm{m}, 3 \sigma$ limit of $0.007 \mathrm{Jy}$ is fitted by 12 and $27.5 \mathrm{~K}$ graybodies with modeled $850 \mu \mathrm{m}$ fluxes of 0.009 and $0.0061 \mathrm{Jy}$, respectively; and for NGC 5353, the observed $850 \mu \mathrm{m}$ flux of $0.017 \mathrm{Jy}$ is fitted by 9 and $27 \mathrm{~K}$ graybodies with modeled $850 \mu \mathrm{m}$ fluxes of 0.011 and $0.006 \mathrm{Jy}$, respectively. The modeled submillimeter fluxes of very cold dust components provide flux estimates of emission that would originate from a second dust component at the respective temperatures and may be consistent with the submillimeter to FIR data. If there is any additional flux contribution to the observed submillimeter flux, the modeled flux from very cold dust components will scale down in proportion to the contributing flux.

Although the two-temperature model may be consistent with the data, it produced relatively high reduced $\chi^{2}$ values of 2, 4.6, 1.6, and 2.2, respectively, for NGC 3962, NGC 4374, NGC 4697, and NGC 5353 (see Table 2), showing that under the fitting criteria assumed in this paper, the single-temperature model of the form in equation (1) fits the presented data slightly better than the two-temperature model. Following this comparison and the fact that the model fits are based on undersampled SEDs with many upper limits, this paper cannot positively identify (or rule out) the presence of cold dust at less than $20 \mathrm{~K}$ in the presented elliptical galaxies.

\section{DERIVED DUST PARAMETERS}

The best-fitting power law plus graybody model to the radio to infrared SEDs constrains the dust to central galactic regions of diameters $\sim 0.4-2.4 \mathrm{kpc}\left(5^{\prime \prime}-9^{\prime \prime}\right)$, average dust temperatures between $\sim 21$ and $28 \mathrm{~K}$, and emissivity index $\beta \simeq 2$ (see Table 2). It was noted in $\S 4$ that when the new submillimeter to ISO FIR data are not considered, such as in the $\chi^{2}$ determinations that do not use the submillimeter to FIR upper limits, it appears that some model fits with $\beta=1.5$ are as good as or better than those with $\beta=2$ (see Table 2). However, it was also noted that for NGC 3962, NGC 4374, and NGC 4697, submillimeter upper limits not included in the $\chi^{2}$ determination fall below the $\beta=1.5$ model plots but are consistent with $\beta=2$ plots, favoring models with $\beta=2$ (see $\S 4)$. Because the size of a dust emitter is inversely proportional to the dust emissivity index and temperature, the diameters derived for $\beta=2$ are upper limits for $\beta$ that range from 1 to 2 and may be larger for dust with temperatures lower than plotted in Figure 2. However, such larger emitting regions would be required to emit flux levels much lower than detected in the presented observations. The dust temperatures around $20 \mathrm{~K}$ and emissivity index $(\beta \approx 2)$, especially, show that dust in elliptical galaxies can exhibit properties similar to those in the Galaxy (cf. dust temperature of 16-21 K and emissivity index of 2; Reach et al. 1995) and probably constitute similar model-predicted amorphous silicate and carbonaceous grains ( $\mathrm{Li} \&$ Draine 2001).

Following Hildebrand (1983), the mass of emitting dust $M_{d}$ can then be estimated from

$$
M_{d}=\frac{S_{\nu} D^{2}}{k_{d} B(\nu, T)},
$$

where $S_{\nu}$ is the measured flux density at frequency $\nu, D$ is the distance to the source, $B(\nu, T)$ is the Planck function, and $k_{d}=3 Q_{\nu} / 4 a \rho$ is the grain mass absorption coefficient where $a$ and $\rho$ are the grain radius and density, respectively. Recently updated values of $k_{d}^{100 \mu \mathrm{m}}=3.5$ and $k_{d}^{60 \mu \mathrm{m}}=10.62 \mathrm{~m}^{2} \mathrm{~kg}^{-1}$
(Li \& Draine 2001) for Galactic-like dust (cf. above) are assumed, yielding dust masses that range from $\sim 1.6 \times 10^{5}$ to $1.9 \times 10^{6} M_{\odot}($ see Table 2$)$.

These dust masses are of similar range as determined in the FIR studies by Roberts et al. (1991) and Goudfrooij \& de Jong (1995), even though those workers used $k_{d}$ values that are typical for dust with emissivity index less than 1.5 (e.g., Hildebrand 1983; Draine 1990) and about 3 times less than the values used here. The difference in the employed $k_{d}$ values is balanced by the fact that dust temperatures derived here are slightly cooler than when estimated from FIR data alone (cf. Roberts et al. 1991; Goudfrooij \& de Jong 1995; Bregman et al. 1998), leading to lower values of $B(\nu, T)$ for this work and the similarity in the dust mass estimates. One advantage of the current study is that, instead of assuming the dust emissivity index and estimating the dust temperatures from the $I R A S 60$ and $100 \mu \mathrm{m}$ flux ratio (cf. Roberts et al. 1991; Goudfrooij \& de Jong 1995), the emissivity index and dust temperatures have been constrained by new FIR and submillimeter to millimeter data that extend to longer wavelengths than earlier IRAS observations and better sample the RayleighJeans tail of the cold dust thermal spectrum. As noted earlier, the resulting constraints suggest that dust in elliptical galaxies has similar properties to Galactic dust.

Derived parameters for NGC 2986 have relatively high uncertainties that are propagated from the IRAS and SCUBA data. IRAS data that were reprocessed with HIRES and SCANPI routines at the NASA/IPAC Center indicate that this source's IRAS flux is probably contaminated by a foreground or background object.

For the two galaxies NGC 3962 and NGC 4374, for which the respective $10^{4.66}$ and $10^{4.54} M_{\odot}$ dust masses estimated from optical extinction studies are available (Goudfrooij \& de Jong 1995), the dust content determined here is about a magnitude larger than that determined from the optical studies of elliptical galaxies, as observed by Goudfrooij \& de Jong (1995) and Wise \& Silva (1996). Both authors proposed that a diffusely distributed dust component not properly accounted for in the optical studies may be responsible for the extra dust mass determined in the infrared. The diffuse dust would probably be heated by a stellar radiation field that is more dilute than in the central regions and thus would be relatively cold. This is withstanding the fact the dust can also be heated by the collision with electrons in the hot gas, with a contribution of the same order of the photon heating even at large radii (Temi et al. 2003). The data presented here are consistent with emission from dust marginally warmer than that seen in some FIR-bright, nearby spiral galaxies (e.g., Dunne \& Eales 2001) and allow flux and dust mass that are consistent with the data to be estimated for dust cooler than $20 \mathrm{~K}$ (see below) within inner galactic regions.

\subsection{Masses for Dust with $T<20 \mathrm{~K}$}

As above, the mass $M_{d}$ of FIR to submillimeter-emitting dust with temperature of less than $20 \mathrm{~K}$ is determined using equation (3), and for the two-temperature dust-fitting results, assuming values of $k_{d}^{450 \mu \mathrm{m}}=0.157$ and $k_{d}^{850 \mu \mathrm{m}}=0.050 \mathrm{~m}^{2}$ $\mathrm{kg}^{-1}$ (Li \& Draine 2001). For (1) NGC 5353, $T=9 \mathrm{~K}$ and $S_{\nu}^{850 \mu \mathrm{m}}=0.011 \mathrm{Jy}$ yield $1.61 \times 10^{4} M_{\odot} ;(2) \mathrm{NGC} 4697, T=$ $12 \mathrm{~K}$ and $S_{\nu}^{850 \mu \mathrm{m}}=0.00087 \mathrm{Jy}$ yield $8.31 \times 10^{4} M_{\odot}$; (3) NGC $4374, T=12 \mathrm{~K}$ and $S_{\nu}^{450 \mu \mathrm{m}}=0.086 \mathrm{Jy}$ yield $6.87 \times 10^{5} M_{\odot}$; and (4) NGC 3962, $T=12 \mathrm{~K}$ and $S_{\nu}^{850 \mu \mathrm{m}}=0.0012 \mathrm{Jy}$ yield $2.71 \times 10^{5} M_{\odot}$. The fluxes used assume the only contribution 
to the detections is the "very cold" and "cold" dust, which may not be the case.

The $12 \mathrm{~K}$ dust mass estimate for NGC 4374 is of the same order as the warmer $28 \mathrm{~K}$ dust mass determined for this galaxy. Therefore, if the $450 \mu \mathrm{m}$ flux for this object is primarily from very cold dust, this component could be as significant as that from the $28 \mathrm{~K}$ dust. For this to be the case, the highfrequency, nonthermal power law would have to turn over or cut off before the $450 \mu \mathrm{m}$ flux. The $12 \mathrm{~K}$ dust mass estimate for NGC 3962 is also of the same order as the warmer $24 \mathrm{~K}$ dust mass determined for this galaxy. In this case, this is all that can be said because only upper limit fluxes are available in the submillimeter, and no radio observations have been made to constrain any low-frequency flux origin for this galaxy. For NGC 5353 and NGC 4697, the 9 K dust mass estimate is about 2 orders of magnitude less than the warmer $28 \mathrm{~K}$, and the $12 \mathrm{~K}$ dust mass estimate is about an order of magnitude less than the warmer $25 \mathrm{~K}$ dust masses determined for these galaxies, respectively. Therefore, in these cases the cooler components, although of relatively considerable mass on their own, do not appear to be as significant as the warmer components within the central galactic regions.

\subsection{Implications of the Fitted Dust Parameters}

It is very interesting that the best-fitting graybody model parameters require dust emitting in the millimeter to FIR to be centrally concentrated and less extended than the stellar component of elliptical galaxies. Because red giant stars lose dusty gas to the ISM, if dust in elliptical galaxies derives from these stars, the dust and stellar spatial distributions would be expected to be cospatial. However, assuming that the dust is indeed produced by stars, as gas cools down and falls into the potential well, distributed dust may drift toward the galactic centers and settle into a central disk. Models of centrally concentrated, distributed dust and the transfer of stellar radiation in elliptical galaxies by Witt et al. (1992) show that dust in such geometry would not affect the observed $r^{(1 / 4)}$ surface brightness law (the de Vaucouleurs profile) caused by the stellar radiation. Moreover, such a distribution of dust would produce and naturally explain the central reddening (or a color gradient that changes with radius) that is observed in elliptical galaxies and cannot be completely explained by models that consider only evolutionary stellar population synthesis and metallicities (e.g., Peletier et al. 1990).

Recent dynamical modeling of dusty gas ejected from evolved stars in elliptical galaxies (or cD galaxies) supports a stellar origin of the central dust clouds seen in these galaxies (Mathews \& Brighenti 2003). The models show that even after entering the hot X-ray-emitting gas, rapid cooling by thermal collisions with dust grains, especially in the central regions, can be faster than the dynamical time in the galactic potential or grain sputtering time, ensuring that some dust survives even in elliptical galaxies with X-ray-emitting gas. Observations indeed show that some dust exists in elliptical galaxies, and as noted by Knapp (1999), the dust probably suggests that elliptical galaxies host modest star formation.

\section{CONCLUSIONS}

The current observations have detected centrally concentrated dust in elliptical galaxies with properties similar to those in the Galaxy (e.g., Reach et al. 1995) and other nearby FIR-bright galaxies (e.g., Dunne \& Eales 2001). Singletemperature, graybody-fitting results imply the dust is slightly cooler than when estimated from FIR data alone; however, dust masses determined using updated dust absorption coefficients ( $\mathrm{Li} \&$ Draine 2001) for Galactic-type dust yield masses similar to estimates from the FIR studies (e.g., Roberts et al. 1991; Goudfrooij \& de Jong 1995). Tentative determinations have been made of flux and mass estimates for dust cooler than $20 \mathrm{~K}$, within the central galactic regions. Resolved submillimeter to FIR observations show a galactic dust temperature decrease from the center to the outer regions (e.g., Leeuw et al. 2002), where the radiation field heating the dust is dilute; and in elliptical galaxies the colder dust is most probably cool, diffusely distributed, and heated by both the dilute stellar radiation and the collision with electrons in the hot gas (Temi et al. 2003). Because of the general paucity of the dust and the observation that it may be centrally concentrated (see above), the direct detection of diffuse, extended dust in elliptical galaxies is challenging. If such diffuse dust with low surface brightness exists, observationally constraining its physical properties may be possible with deeper SCUBA observations (especially mapping) or soon to be commissioned or built sensitive instruments like the Spitzer Space Telescope, SCUBA2, and ALMA.

L. L. Leeuw is supported by NASA grant NAG5-9376. M. H. is supported by the Nordrhein-Westfälische Akademie der Wissenschaften, funded by the Federal State and the Federal Republic of Germany.
Baes, M., \& Dejonghe, H. 2002, MNRAS, 335, 441

Bendo, G. J., et al. 2002, AJ, 123, 3067

Bower, G. A., Heckman, T. M., Wilson, A. S., \& Richstone, D. O. 1997, ApJ, 483, L33

Bregman, J. N., Snider, B. A., Grego, L., \& Cox, C. V. 1998, ApJ, 499, 670 de Vaucouleurs, G., de Vaucouleurs, A., Corwin, H. G., Buta, R. J., Paturel, G., \& Fouqué, P. 1991, Third Reference Catalogue of Bright Galaxies (New York: Springer)

Draine, B. T. 1990, in The Interstellar Medium in Galaxies, ed. H. A. Thronson \& J. M. Shull (Dordrecht: Kluwer), 483

Duncan, W. D., Sandell, G., Robson, E. I., Ade, P. A. R., \& Griffin, M. J. 1990, MNRAS, 243, 126

Dunne, L., \& Eales, S. A. 2001, MNRAS, 327, 697

Fich, M., \& Hodge, P. 1993, ApJ, 415, 75

Goudfrooij, P., \& de Jong, T. 1995, A\&A, 298, 784

Goudfrooij, P., de Jong, T., Hansen, L., \& Norgaard-Nielsen, H. U. 1994, MNRAS, 271, 833

Haas, M. 1998, A\&A, 337, L1
REFERENCES

Haas, M., Müller, S. A. H., Chini, R., Meisenheimer, K., Klaas, U., Lemke, D., Kreysa, E., \& Camenzind, M. 2000, A\&A, 354, 453

Hildebrand, R. H. 1983, QJRAS, 24, 267

Holland, W. S., et al. 1999, MNRAS, 303, 659

Jenness, T., Stevens, J. A., Archibald, E. N., Economou, F., Jessop, N. E., \& Robson, E. I. 2002, MNRAS, 336, 14

Kessler, M. F., Müller, T. G., Arviset, C., García-Lario, P., \& Prusti, T. 2000, ISO: Mission and Satellite Overview, Vol. 1 (GEN) SAI-2000-035/Dc (ver. 2.0; Villafranca: VILSPA), http://www.iso.vilspa.esa.es/manuals/HANDBOOK/ gen_hb

Klaas, U., et al. 2001, A\&A, 379, 823

Knapp, G. R. 1999, in ASP Conf. Ser. 163, Star Formation in Early-Type Galaxies, ed. P. Carral \& J. Cepa (San Francisco: ASP), 119

Knapp, G. R., \& Patten, B. M. 1991, AJ, 101, 1609

Laureijs, R., \& Klaas, U. 1999, ISOPHOT Error Budgets, ISO Explanatory Library Document, SAI/98-091/Dc (ver. 1; Noordwijk: ESA)

Leeuw, L. L., Hawarden, T. G., Matthews, H. E., Robson, E. I., \& Eckart, A. 2002, ApJ, 565, 131 
Leeuw, L. L., Sansom, A. E., \& Robson, E. I. 2000, MNRAS, 311, 683 Lemke, D., et al. 1996, A\&A, 315, L64

Li, A., \& Draine, B. T. 2001, ApJ, 554, 778

Mathews, W. G., \& Brighenti, F. 2003, ApJ, 590, L5

Peletier, R. F., Valentijn, E. A., \& Jameson, R. F. 1990, A\&A, 233, 62

Reach, W. T., et al. 1995, ApJ, 451, 188

Roberts, M. S., Hogg, D. E., Bregman, J. N., Forman, W. R., \& Jones, C. 1991, ApJS, 75, 751

Sandell, G., Jessop, N., \& Jenness, T. 1998, The SCUBA Mapping Cookbook (Hilo: JAC)
Temi, P., Brighenti, F., Mathews, W. G., \& Bregman, J. D. 2004, ApJS, 151, 237

Temi, P., Mathews, W. G., Brighenti, F., \& Bregman, J. D. 2003, ApJ, 585, L121

van Dokkum, P. G., \& Franx, M. 1995, AJ, 110, 2027

Wise, M. W., \& Silva, D. R. 1996, ApJ, 461, 155

Witt, A. N., Thronson, H. A., \& Capuano, J. M. 1992, ApJ, 393, 611 